\title{
Video-assisted thoracoscopic surgery pleurectomy: a suitable alternative for treating malignant pleural effusions
}

\author{
Laurence P. Diggs ${ }^{1}$, Sean P. Martin ${ }^{1}$, Justin A. Drake ${ }^{1}$, R. Taylor Ripley ${ }^{2}$ \\ ${ }^{1}$ Surgical Oncology Program, Center for Cancer Research, National Cancer Institute, National Institutes of Health, Bethesda, MD, USA; ${ }^{2}$ Michael E. \\ DeBakey Department of Surgery, Baylor College of Medicine, Houston, TX, USA \\ Contributions: (I) Conception and design: LP Diggs, RT Ripley; (II) Administrative support: RT Ripley; (III) Provision of study materials or patients: \\ RT Ripley; (IV) Collection and assembly of data: LP Diggs, SP Martin, JA Drake; (V) Data analysis and interpretation: LP Diggs, SP Martin, JA \\ Drake, RT Ripley; (VI) Manuscript writing: All authors; (VII) Final approval of manuscript: All authors. \\ Correspondence to: Laurence P. Diggs, MD. Center for Cancer Research, National Cancer Institute, Building 10, Room 3B-44, Bethesda, MD 20892, \\ USA. Email: laurence.diggs@gmail.com.
}

Background: Malignant pleural effusions (MPEs) are common manifestations of metastatic cancers and
are associated with a dismal prognosis. Talc pleurodesis has been proven to be effective in the management of
MPEs, however, class-action lawsuits linking talc to ovarian adenocarcinoma have rendered it unavailable at
many institutions. As a result, surgeons have resorted to less effective chemical pleurodesis as an alternative
to indwelling pleural drainage catheters. Given the absence of talc, we explored the effectiveness of video-
assisted thoracoscopic surgery (VATS) partial pleurectomy (VPP) for treating MPEs.
Methods: We performed a retrospective review of patients with MPEs managed after talc became
unavailable at our institution. Between 2016 and 2018, we identified five patients who refused pleural
drainage catheters and underwent VPP. Symptoms at presentation included fatigue, dyspnea, and pleuritic
chest pain. All had unilateral MPEs (left n=3, right n=2). VPP included removal of parietal surfaces of the
pleura other than the pleura overlying the subclavian vessels, the mediastinum, and the lung viscera.
Results: There were no significant perioperative adverse events and post-operative pain was well
controlled. Chest tubes were removed between post-operative day (POD) 3 and 7 . Follow-up time ranged
from four to 36 weeks. All patients had symptomatic relief and radiographic evidence of improved MPEs.
No patients required re-interventions. One patient expired six months after surgery while the remaining four
were alive at last follow-up.

Conclusions: VPP offers an effective alternative to chemical pleurodesis for managing MPEs in patients who prefer to avoid pleural drainage catheters.

Keywords: Malignant pleural effusion (MPE); VATS partial pleurectomy (VPP); Talc; Tunneled pleural catheter (TPC)

Submitted Oct 03, 2019. Accepted for publication Feb 05, 2020.

doi: $10.21037 /$ jtd-19-3261

View this article at: http://dx.doi.org/10.21037/jtd-19-3261

\section{Introduction}

Malignant pleural effusions (MPEs) are common complications of primary or metastatic pleural malignancies. MPEs currently affect approximately 150,000 patients per year in the United States and over one million per year worldwide (1). Approximately $50 \%$ to $65 \%$ of all malignant effusions are secondary to cancers of the lung and breast, however any malignancy that metastasizes to the pleura can result in a MPE. Prognosis associated with this condition is very poor with survival ranging from three to twelve months (2). Patients typically suffer considerably from symptoms including fatigue, pleuritic chest pain, dyspnea on exertion and eventually dyspnea at rest. Life-threatening 
complications such as pneumothorax and pneumonia are also commonly reported. As a result of these morbid conditions, patients not only have a limited life expectancy but also endure a poor quality of life (1-3).

Surgery offers the best management of this condition. However, avoiding morbidity and lengthy hospitalizations is critical given these patients' poor prognosis. Surgical interventions must therefore have a reasonably low expectation of complications or repeated interventions. Tunneled pleural catheters (TPC) are highly effective for managing MPEs and currently represent the most common approach for the care of patients suffering from this condition. While TPCs provide effective control of MPE-associated symptoms and can be placed without surgical intervention (4), patients are required to manage them daily and often find them cumbersome. Likely due to these complaints, as many as $35 \%$ of eligible patients refuse this option. The most commonly utilized and successful alternative intervention for MPE that avoids pleural catheters involves surgical drainage with talcbased, chemical, or mechanical pleurodesis. Video-assisted thoracoscopic surgery (VATS) is utilized for all these procedures. VATS enables interventions such as surgical drainage and partial pleurectomies to be performed without the morbidity of an open thoracotomy or the need for additional incisions as the port sites are utilized for chest tube placement (5).

Historically, parietal pleurectomy via thoracotomy achieved effective pleurodesis, but this procedure was largely abandoned due to the morbidity associated with a thoracotomy. Talc pleurodesis, on the other hand, is a highly effective approach for managing MPEs with success rates as high as $88 \%$, low morbidity, and an infrequent need for additional interventions $(2,3,6,7)$. In recent years, however, several major class action lawsuits based on the suspected association between talc and ovarian adenocarcinoma have resulted in talc shortages and a halt in the production of pharmaceutical grade talc by most manufacturers $(8,9)$.

Talc, a mineral consisting primarily of magnesium, silicon, and oxygen, has historically been reported to contain anthophyllite, tremolite, and serpentine chrysolite asbestos as a result of contamination during the extraction process, most notably in regions where there is a higher content of these minerals $(10,11)$. Asbestos is a well-established carcinogen proven to cause mesothelioma. However, none of these carcinogenic trace minerals have been detected in talc manufactured over the last four decades. Moreover, the evidence for the association between talc and cancer is inconclusive (10-15). Yet the production and supply of talc have plummeted given the concern for legal liability.

With talc largely unavailable, other approaches to the management of MPE are necessary. Unfortunately, while chemical pleurodesis with agents such doxycycline or bleomycin presents an alternative to talc, the success rate is substantially lower, and this approach often require several re-interventions (16-19). In fact, no other approach has been reported to offer equivalent results. The utility of these alternatives therefore remains heavily a debated among thoracic surgeons $(6,19)$. In the absence of talc, we explored the effectiveness of VATS partial pleurectomies (VPP) for treating MPEs in patients who would have otherwise undergone VATS talc pleurodesis.

\section{Methods}

\section{Patient selection}

We performed a retrospective review of patients with MPEs managed at our medical center from 2016-2018 given that talc has not been available here since 2016. This study was exempt from institutional review as data had already been collected under our clinical protocols and patients had already signed consent for their records be studied at our institution. This study was conducted in accordance with the Declaration of Helsinki (as revised in 2013).

Patients were selected from a cohort who refused TPC and needed definitive management of a MPE. All patients were symptomatic and had radiographic evidence of an effusion (Figures 1,2). All effusions were confirmed as malignant using cytopathologic analysis. All patients were offered a TPC but did not want to manage an external device. Given these patients' preference as well as the absence of talc, we opted to perform a VATS partial pleurectomy (VPP) for each of these cases (Figure 3).

\section{Procedure description}

VATS is performed with an initial incision in the $8^{\text {th }}$ intercostal space of the posterior axillary line. A second incision is placed $4-5$ centimeters posterior to the initial port in the same interspace. No additional working ports or incisions are necessary as these two sites are ultimately utilized for the insertion of two chest tubes. The effusions are then drained. The parietal pleura is incised with cautery anteriorly about 2 centimeters lateral to the mammary vessels and posteriorly about 4 centimeters laterally to the 

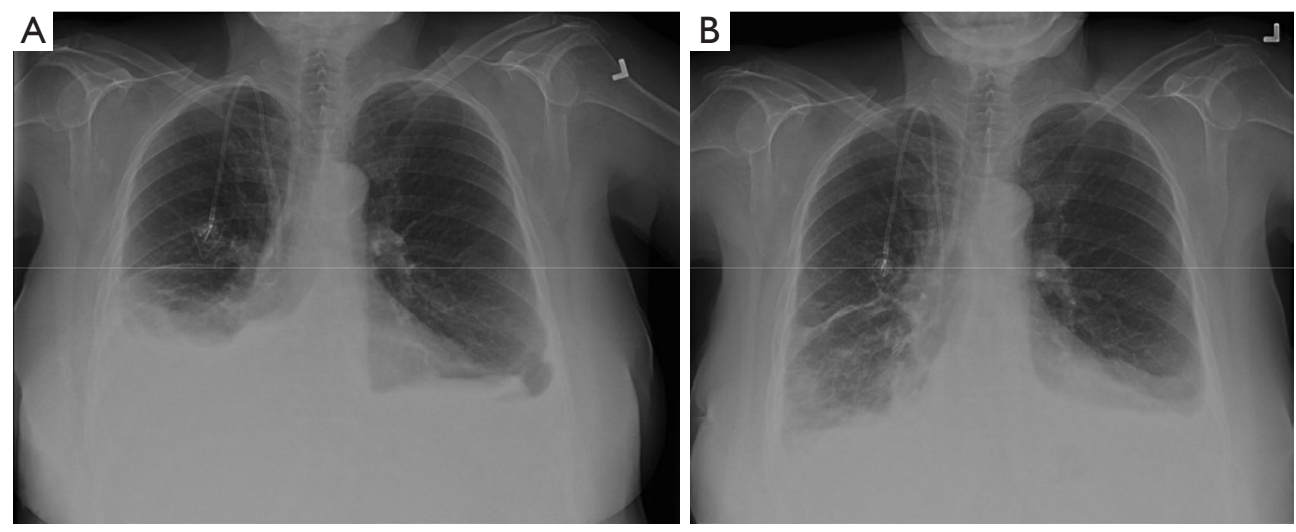

Figure 1 Images before (A) and after (B) VATS partial pleurectomy in a 75-year-old female patient with metastatic breast cancer. VATS, video-assisted thoracoscopic surgery.
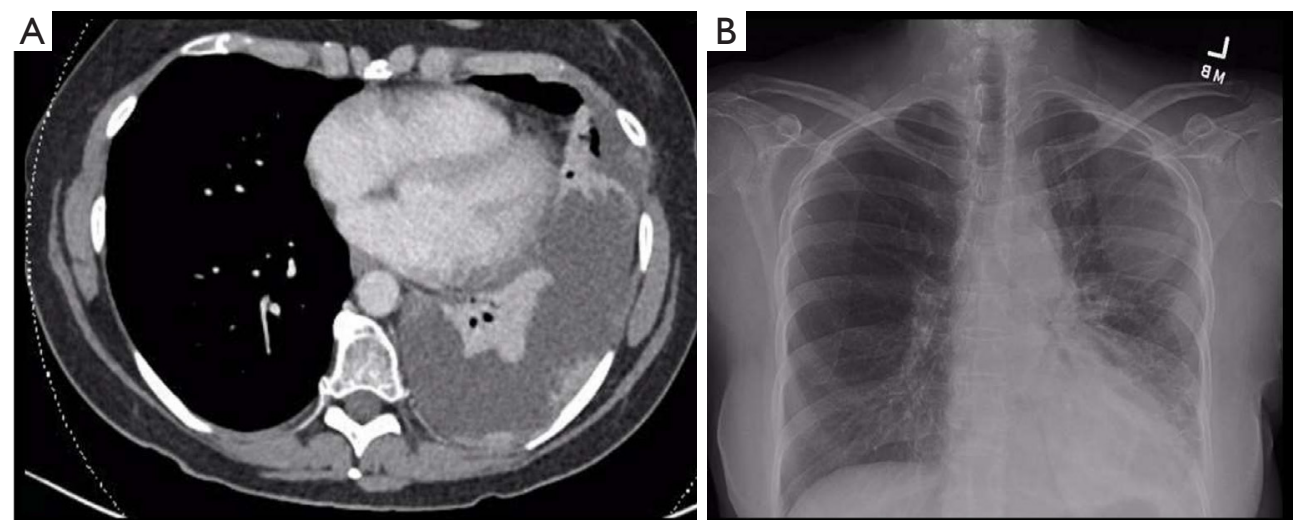

Figure 2 Computed tomography before (A) and chest radiograph after (B) a VATS partial pleurectomy in a 50-year-old female with metastatic pancreatic cancer. VATS, video-assisted thoracoscopic surgery.
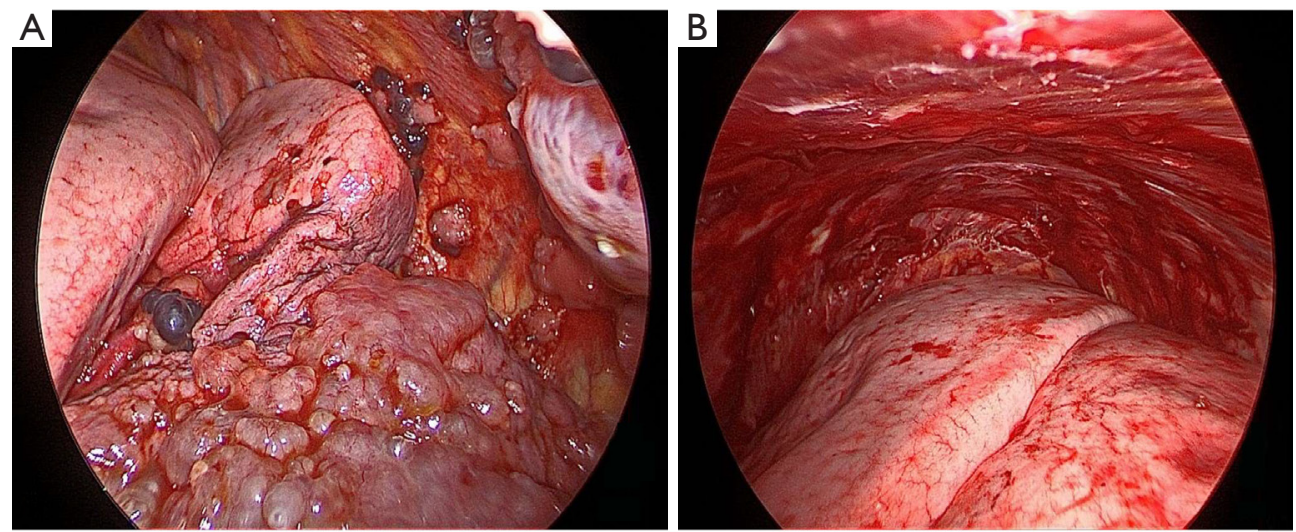

Figure 3 Intraoperative thoracoscopic images of patient before (A) and after (B) VATS partial pleurectomy for metastatic ovarian cancer. VATS, video-assisted thoracoscopic surgery. 
Table 1 Demographic and clinical patient characteristics

\begin{tabular}{|c|c|}
\hline Patient characteristics & Study patients \\
\hline Total patients $(\mathrm{n})$ & 5 \\
\hline \multicolumn{2}{|l|}{ Sex } \\
\hline Female & 4 \\
\hline Male & 1 \\
\hline Mean age (years) & 50.2 \\
\hline \multicolumn{2}{|l|}{ Diagnosis } \\
\hline Pancreatic adenocarcinoma & 1 \\
\hline Renal cell carcinoma & 1 \\
\hline Ductal breast adenocarcinoma & 1 \\
\hline Fallopian tube carcinoma & 1 \\
\hline Synovial carcinoma & 1 \\
\hline Stage 4 & 5 \\
\hline \multicolumn{2}{|l|}{ Pleural effusion laterality } \\
\hline Left & 3 \\
\hline Right & 2 \\
\hline Median time to chest tube removal (POD) & 3 \\
\hline Median follow-up time (weeks) & 6 \\
\hline Alive at last follow up (\%) & 80 \\
\hline
\end{tabular}

vertebral column. These incisions are connected superiorly along the second rib and inferiorly along the $8^{\text {th }}$ and $9^{\text {th }}$ rib. Resection of all parietal pleural surfaces within these incisions is then performed (Figure 3). The pleura overlying the subclavian vessels, the mediastinum, and the pulmonary visceral pleura is not removed. Mechanical pleurodesis with a scratch pad is performed at the apex and on the diaphragm. An intrathoracic regional nerve block from the $2^{\text {nd }}$ to the $10^{\text {th }}$ interspace is performed with $0.25 \%$ Marcaine. Anterior and posterior chest tubes are then inserted using the thoracoscopic port sites. A 0-vicryl suture in the muscle layer tightens the port site around the chest tube to avoid fluid leakage. The chest tubes are placed on suction for 48-72 hours and are removed once the drainage decreases.

\section{Results}

\section{Patient demographics and operative details}

Five patients successfully underwent VPP. The average age of the patients was 50 years (range, $30-75$ years). Four patients were female and one male. Histologies included pancreatic adenocarcinoma $(n=1)$, clear cell renal cell carcinoma $(n=1)$, ductal breast adenocarcinoma $(n=1)$, ovarian carcinoma $(n=1)$, and synovial sarcoma $(n=1)$. During their initial evaluation, these patients reported fatigue, dyspnea, and pleuritic chest pain. Two patients complained of dyspnea on exertion only while the other three reported dyspnea even while at rest. Four patients had undergone previous thoracentesis to control symptoms of their MPEs. All five had unilateral effusions (three left and two right). The procedure was well-tolerated in all cases with no significant peri-operative adverse events. No post-operative pneumothoraxes were noted. The timing of chest tube removal ranged from post-operative day (POD) three to seven (Table 1). No patient needed to have a chest tube replaced. Post-operative pain control required hydromorphone patient-controlled analgesia in four cases for an average of two days (range one to four days) before patients were transitioned to an oral analgesia regimen. One patient was directly started on oral oxycodone and acetaminophen after surgery and required no intravenous analgesia. On average, patients required ten doses (range three to 21 doses) of oxycodone during their hospitalization. All patients were started on a diet on POD0 and ambulated on POD1.

\section{Patient outcomes}

Median post-operative follow-up duration was six weeks (range four to 36 weeks). At follow-up, all patients had radiographic evidence of improved or resolved MPE (Figures 1,2). Every patient also reported significant improvement in symptoms at post-operative follow-up. No patients required re-intervention to manage worsening symptoms or recurrent MPE. One patient expired at six months post-surgery while the remaining four were alive at last follow-up.

\section{Discussion}

Talc pleurodesis and TPCs are two of the most effective and preferred means of managing MPE. The recent shortage of talc for patients who do not want to manage a TPC has resulted in the utilization of non-talc methods often involving alternative chemical pleurodesis. However, such approaches are not nearly as effective as talc (16,19-21). Patients in our study were typically on protocols that 
required precise timing of systemic therapy administration that could not be interrupted by repeated interventions. Therefore, we started performing VATS partial pleurectomy to achieve the highest chance of effective pleurodesis without re-intervention. This approach necessitated an operation and several days in the hospital, but no patients required additional interventions and all participants received systemic therapy without delay.

Insertion of a TPC is the most common approach and should be offered to all patients who may be candidates for VPP. Thomas et al. reported that patients experienced fewer post-operative complications and shorter hospital length of stay with TPC compared to talc (7). Yet, TPCs cause discomfort and require management by the patient, family, and often necessitate home nursing. In addition, the inadequate management of TPC can result in clogging and infections requiring further interventions $(2,3,6)$. Until recently, talc provided an effective alternative to TPC and it remains an optimal means of managing MPEs for many if not most patients who wish to avoid drainage catheters. Despite the reasonable outcomes of VPP, we do not propose that it is superior to talc pleurodesis. Both offer consistent and durable alleviation of MPE symptoms and can be achieved by VATS with low post-operative morbidity. In fact, Rintoul et al. compared outcomes of VATS partial pleurectomy to talc pleurodesis for MPEs and noted no improvement in overall survival or symptom control and longer hospital length of stay and postoperative complications with VPP compared to talc (5). These findings suggest that talc still represents the ideal alternative to TPCs. Yet, as a result of several class action lawsuits, there has been a halt in the production of medical grade talc resulting in its unavailability in most institutions (9).

The specific concerns associated with talc stem from the potential for contamination with asbestos, a highly carcinogenic agent $(8,9)$. However, recent analyses concluded that trace amounts of asbestos have not been identified in talc since the 1970s and that there is no clear association between talc and ovarian adenocarcinoma $(8,11-15,22,23)$. In a cosmetic setting, the carcinogenic risks, no matter how small, may reasonably outweigh the benefits. However, talc constitutes one of the best palliative option available to surgeons caring for patients suffering from effusions associated with their metastatic cancers. Given talc's unproven carcinogenic potential in addition to the poor prognosis and short life expectancy associated with MPEs, we feel that providing this compound for this indication is reasonable and appropriate. Although we cannot issue any legal recommendations and have no expertise in liability, pleural effusions associated with metastatic cancers present a specific clinical setting in which the benefits of talc for its management outweighs the risks. We maintain that VPP nevertheless presents a reasonable approach that may be considered as an alternative first-line treatment of MPE, regardless of the availability of talc.

Our study is not without its limitations. We report data from a single center, in a retrospective manner and we evaluated a small sample size. We are therefore unable to draw broad conclusions or recommendations. In addition, we were unable to perform any comparison between outcomes of patients who had previously been treated for MPE. Our report was aimed at describing a reasonable alternative to TPC or other methods of pleurodesis. Finally, we could not assess hospital length of stay as these patients were typically admitted to other care teams and were admitted for participation in a clinical trial. These patients' discharges were determined by their clinical protocol rather than the resolution of their MPE.

\section{Conclusions}

VATS partial pleurectomy presents thoracic surgeons with a feasible alternative to talc pleurodesis or TPC with minimal morbidity and effective control of MPEs. Further studies may be necessary to establish this approach as a standard of care for selected patients in an era where talc is unavailable. Further discussions may also be warranted on the use of talc in the palliative setting where it may offer the least invasive option. VPP should be considered as part of the decision algorithm for patients with MPE especially if talc is unavailable and patients want to avoid TPC.

\section{Acknowledgments}

Funding: None.

\section{Footnote}

Conflicts of Interest: All authors have completed the ICMJE uniform disclosure form (available at http://dx.doi. org/10.21037/jtd-19-3261). The authors have no conflicts of interest to declare.

Ethical Statement: The authors are accountable for all aspects of the work in ensuring that questions related 
to the accuracy or integrity of any part of the work are appropriately investigated and resolved. This study was exempt from institutional review as data had already been collected under our clinical protocols and patients had already signed consent for their records be studied at our institution. This study was conducted in accordance with the Declaration of Helsinki (as revised in 2013).

Open Access Statement: This is an Open Access article distributed in accordance with the Creative Commons Attribution-NonCommercial-NoDerivs 4.0 International License (CC BY-NC-ND 4.0), which permits the noncommercial replication and distribution of the article with the strict proviso that no changes or edits are made and the original work is properly cited (including links to both the formal publication through the relevant DOI and the license). See: https://creativecommons.org/licenses/by-nc-nd/4.0/.

\section{References}

1. Lee YC, Light RW. Management of malignant pleural effusions. Respirology 2004;9:148-56.

2. Roberts ME, Neville E, Berrisford RG, et al. Management of a malignant pleural effusion: British Thoracic Society Pleural Disease Guideline 2010. Thorax 2010;65 Suppl2:ii32-40.

3. Fitzgerald DB, Koegelenberg CFN, Yasufuku K, et al. Surgical and non-surgical management of malignant pleural effusions. Expert Rev Respir Med 2018;12:15-26.

4. Davies HE, Mishra EK, Kahan BC, et al. Effect of an indwelling pleural catheter vs chest tube and talc pleurodesis for relieving dyspnea in patients with malignant pleural effusion: the TIME2 randomized controlled trial. JAMA 2012;307:2383-9.

5. Rintoul RC, Ritchie AJ, Edwards JG, et al. Efficacy and cost of video-assisted thoracoscopic partial pleurectomy versus talc pleurodesis in patients with malignant pleural mesothelioma (MesoVATS): an open-label, randomised, controlled trial. Lancet 2014;384:1118-27.

6. Azzopardi M, Porcel JM, Keogelenberg CF, et al. Current controversies in the management of malignant pleural effusions. Semin Respir Crit Care Med 2014;35:723-31.

7. Thomas R, Fysh ETH, Smith NA, et al. Effect of an Indwelling Pleural Catheter vs Talc Pleurodesis on Hospitalization Days in Patients With Malignant Pleural Effusion: The AMPLE Randomized Clinical Trial. JAMA 2017;318:1903-12.

8. Muscat JE, Huncharek MS. Perineal talc use and ovarian cancer: a critical review. Eur J Cancer Prev 2008;17:139-46.

9. Hsu T. Johnson \& Johnson Told to Pay \$4.7 Billion in Baby Powder Lawsuit, in The New York Times 2018.

10. Rohl AN, Langer AM, Shelikoff IJ, et al. Consumer talcums and powders: mineral and chemical characterization. J Toxicol Environ Health 1976;2:255-84.

11. Gilbert CR, Furman BR, Feller-Kopman DJ, et al. Description of Particle Size, Distribution, and Behavior of Talc Preparations Commercially Available Within the United States. J Bronchology Interv Pulmonol 2018;25:25-30.

12. Gordon RE, Fitzgerald S, Millette J. Asbestos in commercial cosmetic talcum powder as a cause of mesothelioma in women. Int J Occup Environ Health 2014;20:318-32.

13. Pierce JS, Riordan AS, Miller EW, et al. Evaluation of the presence of asbestos in cosmetic talcum products. Inhal Toxicol 2017;29:443-56.

14. The Lancet Oncol Editorial. When is a carcinogen not a carcinogen? Lancet Oncol 2016;17:681.

15. O'Brien KM, Tworoger SS, Harris HR, et al. Association of powder use in the genital area with risk of ovarian cancer. JAMA 2020;323:49-59.

16. Kuzdzał J, Sladek K, Wasowski D, et al. Talc powder vs doxycycline in the control of malignant pleural effusion: a prospective, randomized trial. Med Sci Monit 2003;9:PI54-9.

17. Patz EF Jr, McAdams HP, Erasmus JJ, et al. Sclerotherapy for malignant pleural effusions: a prospective randomized trial of bleomycin vs doxycycline with small-bore catheter drainage. Chest 1998;113:1305-11.

18. Rafiei R, Yazdani B, Ranjbar SM, et al. Long-term results of pleurodesis in malignant pleural effusions: Doxycycline vs Bleomycin. Adv Biomed Res 2014;3:149.

19. Clive AO, Jones HE, Bhatnager R, et al. Interventions for the management of malignant pleural effusions: a network meta-analysis. Cochrane Database Syst Rev 2016;(5):CD010529.

20. Diacon AH, Wyser C, Bolliger CT, et al. Prospective randomized comparison of thoracoscopic talc poudrage under local anesthesia versus bleomycin instillation for pleurodesis in malignant pleural effusions. Am J Respir Crit Care Med 2000;162:1445-9.

21. Fentiman IS, Rubens RD, Hayward JL. A comparison of intracavitary talc and tetracycline for the control of pleural effusions secondary to breast cancer. Eur J Cancer Clin Oncol 1986;22:1079-81. 
22. Huncharek M, Muscat J. Perineal talc use and ovarian cancer risk: a case study of scientific standards in environmental epidemiology. Eur J Cancer Prev 2011;20:501-7.

Cite this article as: Diggs LP, Martin SP, Drake JA, Ripley RT. Video-assisted thoracoscopic surgery pleurectomy: a suitable alternative for treating malignant pleural effusions. J Thorac Dis 202 1;13(4):2177-2183. doi: 10.21037/jtd-19-3261
23. Finley BL, Benson SM, Marsh GM. Cosmetic talc as a risk factor for pleural mesothelioma: a weight of evidence evaluation of the epidemiology. Inhal Toxicol 2017;29:179-85. 